International Journal of Electronics Engineering Research.

ISSN 0975-6450 Volume 9, Number 4 (2017) pp. 527-543

(C) Research India Publications

https://dx.doi.org/10.37622/IJEER/9.4.2017.527-543

\title{
A Zynq Evaluation and Development board based Z-Quad for Precision Agriculture
}

\author{
${ }^{1}$ Britto Martin Paul N. \\ Research Scholar, Malla Reddy Engineering College, \\ Telangana State, India.
}

${ }^{2}$ Rajendra Prasad K.

Assistant Professor, Department of Electronics and Communications, Malla Reddy Engineering College, Telangana State, India.

${ }^{3}$ Srikanth M.

Project Engineer-1, Centre for Development of Advanced Computing (C-DAC), Hyderabad, Telangana State, India.

\begin{abstract}
In the present generation and for the future generation the Quadcopters will play a vital role in helping human person in doing many works where surveillance and security becomes a primary concern especially in the Agriculture sector. Currently we have quads mostly working on the Arduino controllers and some using the ARM controllers. Taking this a step further I am using Zed (Zynq Evaluation and Development) board in the Quad and so I have named it Z-Quad. The Z-Quad having a Zed board is very much compatible to many on board peripherals of our concern whereas with the other controllers it is very much limited. In this paper I have developed a model that caters to Precision Agriculture. I have used VIVADO platform in the Linux OS and the corresponding SDT (Software Development Tool) in executing the code. The Triple Timer Counters play a vital role in driving the BLDC (Brushless DC) motors.
\end{abstract}

Keywords: Quadcopter; Precision Agriculture; Electronic Speed Controller; Zed Board; Vivado; Triple Timer Counter; Zynq-7000; All Programmable System on Chip; Programmable Logic; Processing System. 


\section{INTRODUCTION}

Z-Quad is based on the Zynq®-7000 family. This family is based on the Xilinx® All Programmable System on Chip (AP SoC) architecture. Thus Z-Quad integrates a feature-rich dual-core ARM ${ }^{\circledR}$ Cortex ${ }^{\mathrm{TM}}$-A9 MPCore ${ }^{\mathrm{TM}}$ based processing system (PS) and Xilinx programmable logic (PL) in a single device, built on a state-of-the-art, high-performance, low-power (HPL), $28 \mathrm{~nm}$, and high-k metal gate (HKMG) process technology. The ARM Cortex-A9 MPCore CPUs are the heart of the PS which also includes on-chip memory, external memory interfaces, and a rich set of I/O peripherals which is very much lacking in the Arduino Uno and ARM Controller units [1].

The estimation of the position is a very challenging task as the contact-type sensors like the rotary encoder cannot be used. Many studies have been made on contactless sensors such as cameras or laser range sensors for self-localization and building maps[2]-[7]. In all the approaches [4][5][6][7] proposed they aim at helping the Quadcopter to navigate and for surveillance only in the indoor environment. The requirement is that we need an accurate and real-time self-localization and mapping method in the outdoor real-time framework without GPS (Global Positioning System).

There are two important facts to be noted. The first is that the Quad becomes large and heavy because of the on-board systems [4][5][6][7]. It also becomes heavy in the sense that current vision-based self-localization and mapping algorithms like PTAM (Parallel Tracking and Mapping) [11] requires much calculation and it becomes a heavy task. The second is that two separate circuit boards are used in most of the systems. First board is used as an embedded Linux control processing unit used for high level calculation. In this image processing and task scheduling is done. Second board is a micro-controller unit board used for low level processing. In this, communication to sensors and actuators is done by calling the functions that are needed to be called and finished in real time framework.

In the similar frame of networking Arduino Uno and ARM controllers were used to address the concerns. Even FPGA technology was also used to address the concern. For low level missions and limited calculations it was sufficient but for the high level missions it was hard for the Arduino Uno and ARM controllers to tackle. The requirement was that it needed high end calculations with rich memory resources. This is very much found in the Xilinx ${ }^{\circledR}$ Zynq ${ }^{\circledR}-7000$ All Programmable System on Chip (AP SoC) architecture. It includes an ARM 9 dual-core CPU and FPGA in onechip. Without using two different circuit boards the dual-core CPUs enable the ZQuad to operate at two different levels of tasks. Furthermore, the embedded FPGA system enables high speed signal processing such as image and sound processing in parallel and sharing memory with two CPUs. The Linux operating system which has been developed in the Zynq community can be installed in one or two of the CPUs. Thus, the Linux-based applications of the Z-Quad can be built with open-source libraries such as OpenCV and Julius in combination. In this paper, we present an approach to build a novel size Linux-based, FPGA-based onboard Z-Quad system with the Zynq-7000 AP SoC. 


\section{ZED BOARD ARCHITECTURE}

The Zed Board of the Zynq ${ }^{\circledR}-7000$ family is based on the Xilinx ${ }^{\circledR}$ All Programmable SoC (AP SoC) architecture as shown in figure 1. This board integrates a feature-rich dual-core $\mathrm{ARM}{ }^{\circledR}$ Cortex $^{\mathrm{TM}}$-A9 MPCore ${ }^{\mathrm{TM}}$ based processing system (PS) and Xilinx programmable logic (PL) in a single device, built on a state-of-the-art, highperformance, low-power (HPL), $28 \mathrm{~nm}$, and high-k metal gate (HKMG) process technology.

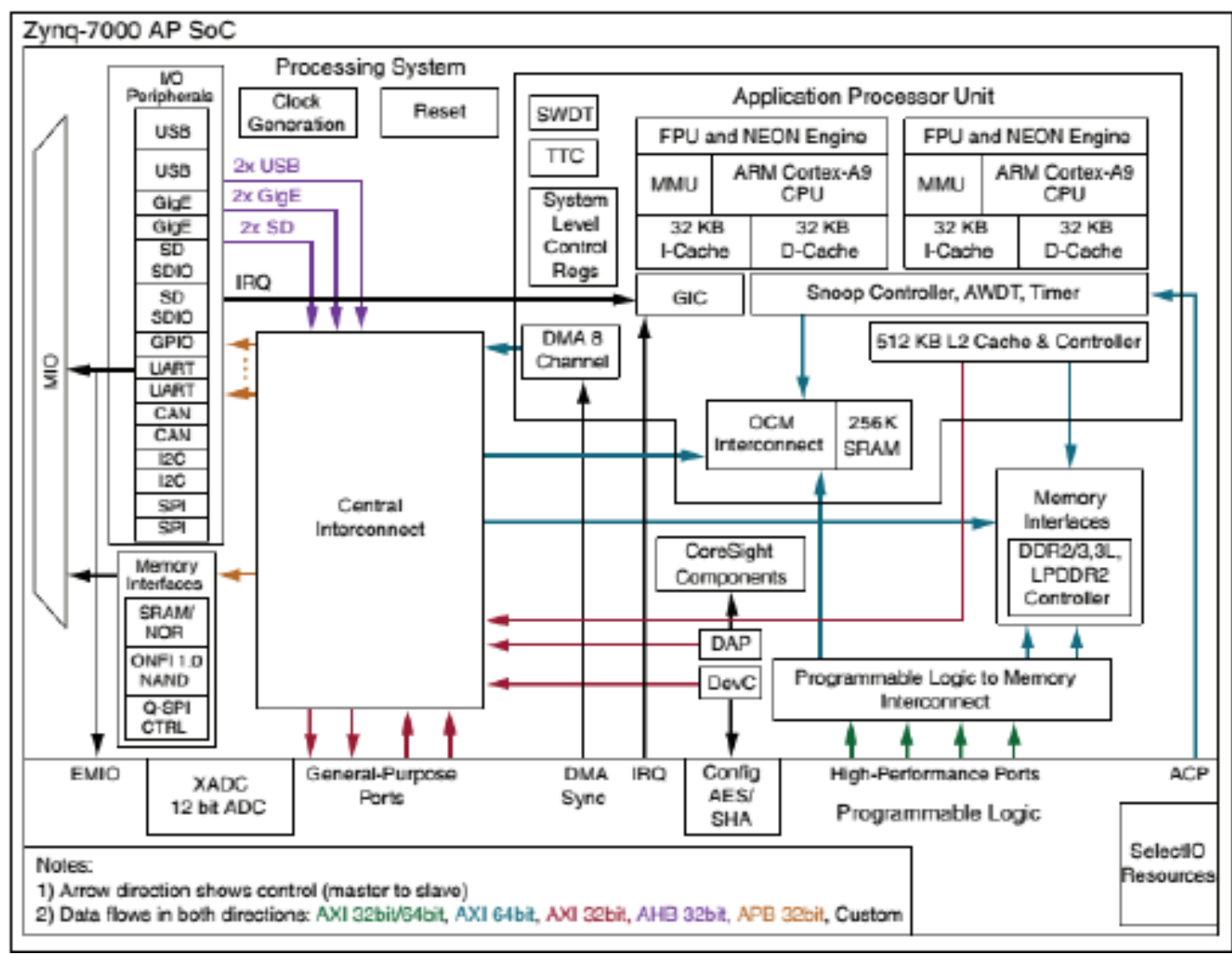

Fig.1: Zynq-7000 AP SoC

The ARM Cortex-A9 MPCore CPUs are the heart of the PS which also includes onchip memory, external memory interfaces, and a rich set of I/O peripherals. The Zed board offers the flexibility and scalability of an FPGA, while providing performance, power, and ease of use typically associated with ASIC and ASSPs. The range of devices in the Zed board of the Zynq-7000 AP SoC family enables designers to target cost-sensitive as well as high-performance applications from a single platform using industry-standard tools. While each device in the Zynq-7000 family contains the same PS, the PL and I/O resources vary between the devices. As a result, the Zed board of the Zynq-7000 AP SoC devices is able to serve a wide range of applications [1]. 


\section{APPLICATIONS OF THE ZED BOARD}

The following are the applications of the Zed Board:

(i) The Zed boards are very much used in automotive driver assistance. This application is very much visible in the Z-Quad. It gives the information of the driver and all the information of the on-board peripherals.

(ii) The Zed board can be used by a camera to broadcast in the real time framework. This application is used very much in the Precision Agriculture so as to get the real time information of the crop and accordingly an action could be taken by the Z-Quad.

(iii) The Zed board is also used in the Industrial motor control especially with regard to the speed of the motor. This aspect is used in the Z-Quad for increasing or decreasing the speed of the Brushless DC Motors so as to change the direction of the Z-Quad through Yaw, Pitch, Throttle and Roll. It is also used in the industrial networking and as a vision of the machine.

(iv) The Zed board is very much in tune with the Internet Protocol. Thus GPS, WIFI and all the smart accessories can be configurable on the Zed board.

(v) The Zed board is compatible with Long-Term Evolution (LTE) standard. It is a standard for high-speed wireless communication for mobile phones and data terminals, based on the GSM/EDGE and UMTS/HSPA technologies. It increases the capacity and speed using a different radio interface together with core network improvements.

(vi) The Zed boards are applicable in the area of Medical Diagnostics and imaging.

(vii) Generally Printers perform a single function but through the Zed boards the Printers perform many functions.

(viii) The Video and the night vision equipment are interfaced easily with the Zed board.

The Zynq-7000 architecture of the Zed board conveniently maps the custom logic and software in the PL and PS respectively. It enables the realization of unique and differentiated system functions. The integration of the PS with the PL provides levels of performance that two-chip solutions (for example, an ASSP with an FPGA) cannot match due to their limited I/O bandwidth, loose-coupling and power budgets. Xilinx and the Xilinx Alliance partners offer a large number of soft IP modules for the Zynq7000 family. Stand-alone and Linux device drivers are available for the peripherals in the PS and the PL from Xilinx and additional OSes and board support packages (BSPs) from partners. The award-winning ISE® Design Suite: Embedded Edition development environment enables a rapid product development for software, hardware, and systems engineers. Many third-party software development tools are also available [1]. 


\section{Z-QUAD ARCHITECTURE}

The Architecture of Z-Quad consists of three main features as shown in figure 2.

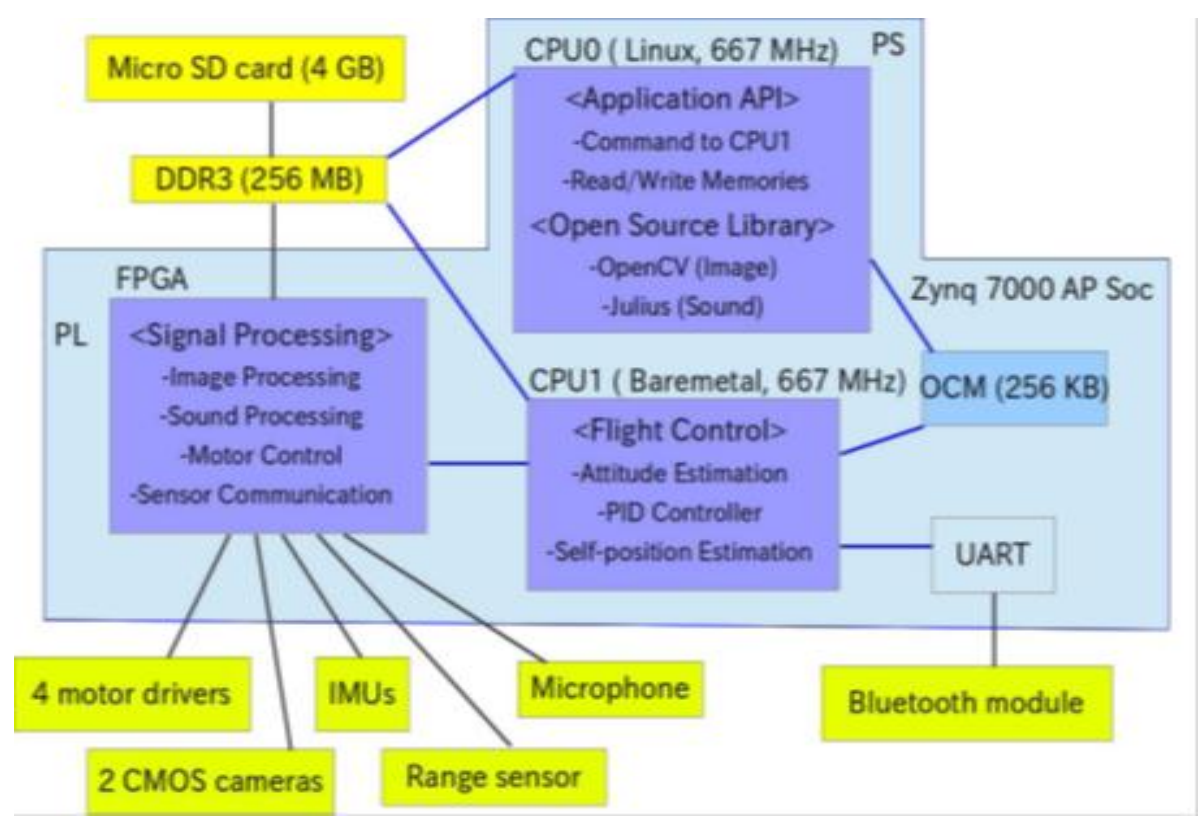

Fig 2: Z-Quad Overview

The First is that, the Z-Quad is pre-built with the Linux-based software development tools. The commands for flying applications are written by the users in $\mathrm{C}$ language by themselves and compilation is done using the library programs in the Z-Quad. They can also combine their own program with an open-source library of image processing and sound recognition engine. All programs are loaded to the Zynq chip from a single micro SD card, in which the Linux file system including the user's programs exists. For this reason, building IDE (Integrated Development Environment) and installing a writer program to send a binary stream to the external flash memory is not required with a host computer.

The second is that FPGA technology enables fast and efficient signal processing. The Z-Quad system has two CMOS cameras and PDM (Pulse Density Modulation) microphone, and both of them require strict timing to be processed correctly. FPGA makes it possible to preprocess images and sound. Thus the resources of the dual-core CPUs can be used very efficiently.

The third is that Z-Quad is the only quad operational using the Zed board. It comes installed with the Linux operating system and is able to fly autonomously with an onboard intelligence by estimating its position in the real-time. The high processing speed and its high end calculations support it to take up missions that are very typical in Precision Agriculture. 


\section{HARDWARE COMPONENTS}

5.1 Zed Board: This is the heart and soul of the Z-Quad as shown in figure 3.

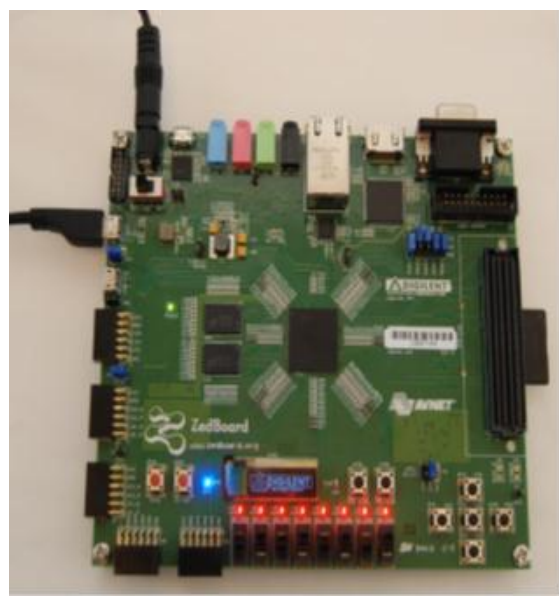

Fig 3: Zed board

The following are its features:

5.1.1 Processor -Zynq ${ }^{\mathrm{TM}}-7000$ All Programmable (AP) System on Chip (SoC) XC7Z020-CLG484-1.

5.1.2 Memory-512 MB DDR3, 256 Mb Quad-SPI Flash and 4 GB SD card.

5.1.3 Communication-Onboard USB-JTAG Programming, 10/100/1000 Ethernet, USB OTG 2.0 and USB-UART.

5.1.4 Expansion connectors- FMC-LPC connector (68 single-ended or 34 differential I/Os), 5 Pmod $^{\mathrm{TM}}$ compatible headers $(2 \times 6)$ and Agile Mixed Signaling (AMS) header.

5.1.5 Clocking- 33.33333 MHz clock source for PS and 100 MHz oscillator for PL.

5.1.6 Display-HDMI output supporting 1080p60 with 16-bit, YCbCr, 4:2:2 mode color, VGA output (12-bit resolution color) and 128x32 OLED display.

5.1.7 Configuration and Debug-Onboard USB-JTAG interface and Xilinx Platform Cable JTAG connector.

5.1.8 General Purpose I/O-8 user LEDs, 7 push buttons, and 8 DIP switches.

5.2 BLDC (3000mAh, 25C): Brushless DC electric motor also known as electronically commutated motors are synchronous motors that are powered by a DC electric source via integrated inverter/switching power supply, which produces an AC electric signal to drive the motor. A BLDC motor for quadcopter is constructed with a permanent magnet rotor and wire wound stator poles. 
5.3 ESC: Electronic Speed Controller (ESC) is used to control BLDC motor. It takes signal from microcontroller of the Zed board and breaks into three parts and sends it to the BLDC motor. We would require four ESCs as we are using four BLDC motors. The ESC is an inexpensive motor controller board that has a battery input and a three phase output for the motor. Each ESC is controlled independently by a PPM signal (similar to PWM). The frequency of the signals vary, but for a Quadcopter it is recommended the controller should support high enough frequency signal, so the motor speeds can be adjusted quick enough for optimal stability.

5.4 Accelerometer Sensor: This sensor is a digital-output triple-axis accelerometer with a programmable full scale range of $\pm 2 g, \pm 4 g, \pm 8 g$ and $\pm 16 g$. The integrated 16bit ADCs enable simultaneous sampling of accelerometers while requiring no external multiplexer. The Accelerometer normal operating current is $500 \mu \mathrm{A}$. The Low power accelerometer mode current is $10 \mu \mathrm{A}$ at $1.25 \mathrm{~Hz}, 20 \mu \mathrm{A}$ at $5 \mathrm{~Hz}, 60 \mu \mathrm{A}$ at $20 \mathrm{~Hz}$, and $110 \mu \mathrm{A}$ at $40 \mathrm{~Hz}$. It has a good orientation detection and signaling, Tap detection, Userprogrammable interrupts, High-G interrupt and User self-test.

5.5 Gyroscope Sensor: This Sensor has Digital-output X-, Y-, and Z-Axis angular rate sensors (gyroscopes) with a user-programmable full scale Range of $\pm 250, \pm 500$, \pm 1000 , and $\pm 2000^{\circ} / \mathrm{sec}$. The External sync signal connected to the FSYNC pin supports image, video and GPS synchronization. The Integrated 16-bit ADCs enable simultaneous sampling of gyros. It has Enhanced bias and sensitivity temperature stability which reduces the need for user calibration Improved low-frequency noise performance. It has Digitally-programmable low-pass filter. The Gyroscope operating current is $3.6 \mathrm{~mA}$. Its standby current is $5 \mu \mathrm{A}$. It has a Factory calibrated sensitivity scale factor. It has the provision for User self-tests.

5.6 Radio receiver: This receives $2.4 \mathrm{GHz}$ signals coming from the transmitter side. It has got six independent channels to receive the signal from the transmitter and then send the signal to the microcontroller for further processing. Its current consumption is less than $40 \mathrm{~mA}$ and works on 5 volt power supply.

5.7 LiPO Battery: Lithium batteries are the preferred power sources for most electric modellers today. They offer high discharge rates and a high energy storage/weight ratio. However, using them properly and charging them correctly is no trivial task.

5.8 DC Voltage Supply: A power supply is an electronic device that supplies electric energy to an electrical load. The primary function of a power supply is to convert one form of electrical energy to another and, as a result, power supplies are sometimes referred to as electric power. 30 RPM Side Shaft 37mm Diameter Compact DC Gear Motor is suitable for small robots automation systems. It has sturdy construction with gear box built to handle stall torque produced by the motor. Drive shaft is supported from both sides with metal bushes. Motor runs smoothly from $4 \mathrm{~V}$ to $12 \mathrm{~V}$ and gives 30 
$\mathrm{RPM}$ at $12 \mathrm{~V}$. Motor has $6 \mathrm{~mm}$ diameter, $22 \mathrm{~mm}$ length drive shaft with $\mathrm{D}$ shape for excellent coupling with internal hole. It weighs $125 \mathrm{gm}$.

5.9 Camera: Video input is generated by the VITA-2000 image sensor from ON Semiconductor, which is configured for 1080p60 resolution. The raw Bayer subsampled image is converted to an RGB image by an image processing pipeline implemented using LogiCORETM IP video cores that remove defective pixels, demosaic, and color-correct the image. A video frame buffer is Implemented in the processing system (PS) DDR3 memory, making images accessible to the ARM ${ }^{\circledR}$ processor cores via the AXI Video Direct Memory Access (VDMA). The video frame buffer is not required for the operation of the image processing pipeline, but is included in the design to enable the capture of input video images for analysis[8].

\section{SOFTWARE COMPONENTS}

In this part we need to create a Zynq-7000 processor based design and instantiate IP in the processing logic fabric (PL) to complete our design. Then we need to take the design through implementation, generate a bitstream, and export the hardware to Software Development kit (SDK).

The following is needed in order to proceed further:

- $\quad$ Vivado w/ Xilinx SDK (tested, version 2013.2)

- $\quad$ Zed board (tested, version D)

There are two parts in this they are: The first part deals with Building a Zynq-7000 Processor Hardware. It has the following steps namely:

Step 1: Start the Vivado IDE and Create a Project

Step 2: Create an IP Integrator Design

Step 3: Customize Instantiated IP and Use Block Designer Assistance

Step 4: Generate HDL Design Files

Step 5: Implement Design and Generate Bitstream

Step 6: Export Hardware to SDK

The second part deals with Building a Zynq-7000 Processor Software. It has the following steps namely:

Step 1: Start SDK and Create a Software Application

Step 2: Run the Software Application-In this step we also need to add a Breakpoint.

Step 3: Executing the Software [9]

6.1 Linux system: There are two steps involved over here. The first is to launch a serial console programme. The second step is the Users login to the Linux operating system. This will establish connection to a Bluetooth module. This module is 
connected to a UART module in the PS system of Zynq. There is no need to install a cross-compile development and writer program to user's host computer since tools for building programs are already installed in the on-board Linux system. The Linux kernel image and root file systems (Ubuntu12.11) are provided by Linaro. The software library is coded for flying applications in Linux in Embedded C language so that users can make their own program combined with other open-source libraries. The software library consists of low level communication with CPU1 and the PL system by reading or writing the content in OCM and DDR3. The following operations are supported in the software library.

- The four states of the quadcopter (going up, hovering, going down and stop)

- Setting desired roll, pitch, and yaw angle.

- Setting desired position and gain on basis of self-position estimation.

- Reading the image and sound data.

- Calibrating flying parameters and bias of thrusts.

Image processing and sound recognition are possible by compiling programs with OpenCV or Julius library.

6.2 Baremetal System: The baremetal system runs on CPU1. The stability in the control unit of the quadcopter is achieved by calling the sensing and the actuating functions at small fixed intervals. The self-position estimation of the Z-Quad is very much needed. It is required especially in the real time for on-board and autonomous hovering. These functions are difficult to be run on the Linux System. Here the Baremetal system plays a vital role. In this system CPU1 does two main functions. The first one is to call a function periodically and the second is the loop function.

6.3 Triple Timer Counter (TTC): The TTC contains three independent timers/counters as shown in figure 4.

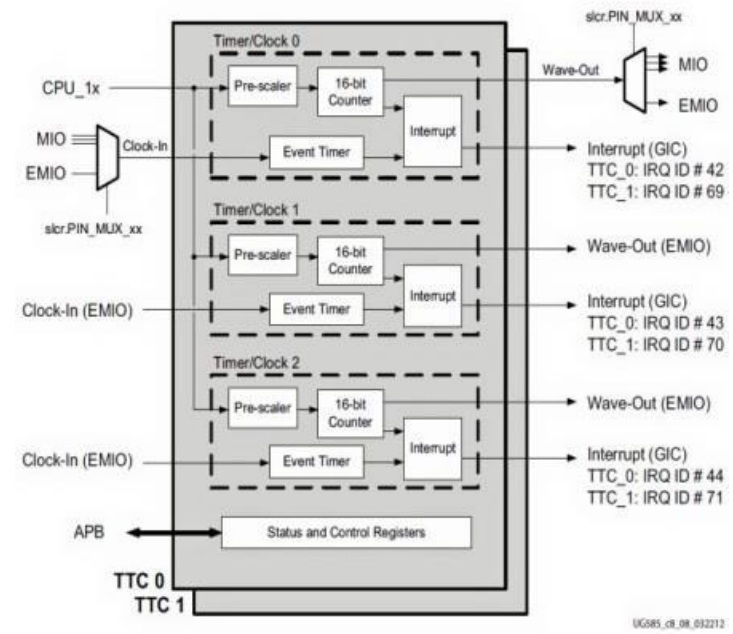

Fig 4: Triple Timer Counter 
There are two TTC modules in the PS, for a total of six timers/counters. TTC1 controller can be configured for secure or non-secure mode using the nic301_addr_region_ctrl_registers.security_apb [ttc1_apb] register bit. The three timers within a TTC controller have the same security state.

Each of the triple timer counters has:

(i) Three independent 16-bit prescalers and 16-bit up/down counters.

(ii) Selectable clock input from: Internal PS bus clock (CPU_1x), Internal clock (from PL), External clock (from MIO).

(iii) Three interrupts, one for each counter.

(iv) Interrupt on overflow, at regular interval, or counter matching programmable values.

(v) Generates waveform output (for example, PWM) through the MIO and to the PL.

Each prescaler module can be independently programmed to use the PS internal bus clock (CPU_1x), or an external clock from the MIO or the PL. For an external clock, SLCR registers determine the exact pinout through the MIO or from the PL. The selected clock is then divided down from $/ 2$ to $/ 65536$, before being applied to the counter. The counter module can count up or count down, and can be configured to count for a given interval. It also compares three match registers to the counter value, and generate an interrupt if one matches.

The interrupt module combines interrupts of various types: counter interval, counter matches, counter overflow, event timer overflow. Each type can be individually enabled. Each counter module can be independently programmed to operate in either of the following two modes:

6.3.1 Interval mode: The counter increments or decrements continuously between 0 and the value of the Interval register, with the direction of counting determined by the DEC bit of the Counter Control register. An interval interrupt is generated when the counter passes through zero. The corresponding match interrupt is generated when the counter value equals one of the Match registers.

6.3.2 Overflow mode: The counter increments or decrements continuously between 0 and 0xFFFF, with the direction of counting determined by the DEC bit of the Counter Control register. An overflow interrupt is generated when the counter passes through zero. The corresponding match interrupt is generated when the counter value equals one of the Match registers [1].

In the Z-Quad the triple timer counter plays a vital role as it is very much used in actuating the values from the gyro and the accelerometer. The Pitch, Yaw, Throttle and Roll are generated through the triple timer counters in the Z-Quad. In the implementation process the testing through triple timer counters was successful. 


\subsubsection{Clock Input Option for Counter/Timer}

The following shows how AP SoC selects the clock source for TTC0 counter/timer 0:

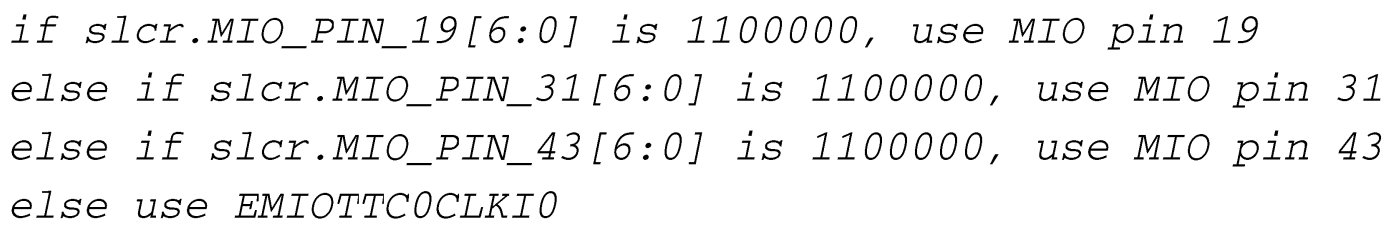

TTC0 counter/timer 1 can use only EMIOTTCOCLKI1.

TTC0 counter/timer 2 can use only EMIOTTCOCLKI2.

The following shows how Zynq SoC selects the clock source for TTC1 counter/timer 0 :

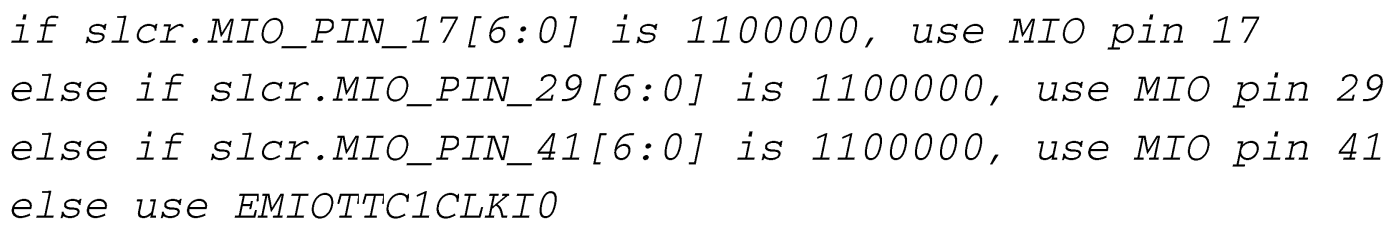

TTC1 counter/timer 1 can use only EMIOTTC1CLKI1.

TTC1 counter/timer 2 can use only EMIOTTC1CLKI2.

It is important to note when an MIO pin or EMIOTTCxCLKIx is chosen to be the clock source, if the clock stops running, the corresponding Count Value register retains the old value, regardless of the fact that the clock has already stopped [1].

\section{IMPLEMENTATION PROCESS}

With all the hardware and software components available the building of the Z-Quad based on the Zynq-7000 AP SoC processor was indeed a difficult task. At each and every moment testing had to be done to acquire the desired values. The following are the steps involved in making of the Z-Quad:

Step 1: Interfacing the components to the Zed board. This was a herculean task as the board contains both the FPGA as well as the ARM processor. At first the software through Ubuntu OS had to be configured using the FPGA in the PL section of the Zed board. After the formal Hello World programme is run successfully the following happens as shown in the figure 5. 


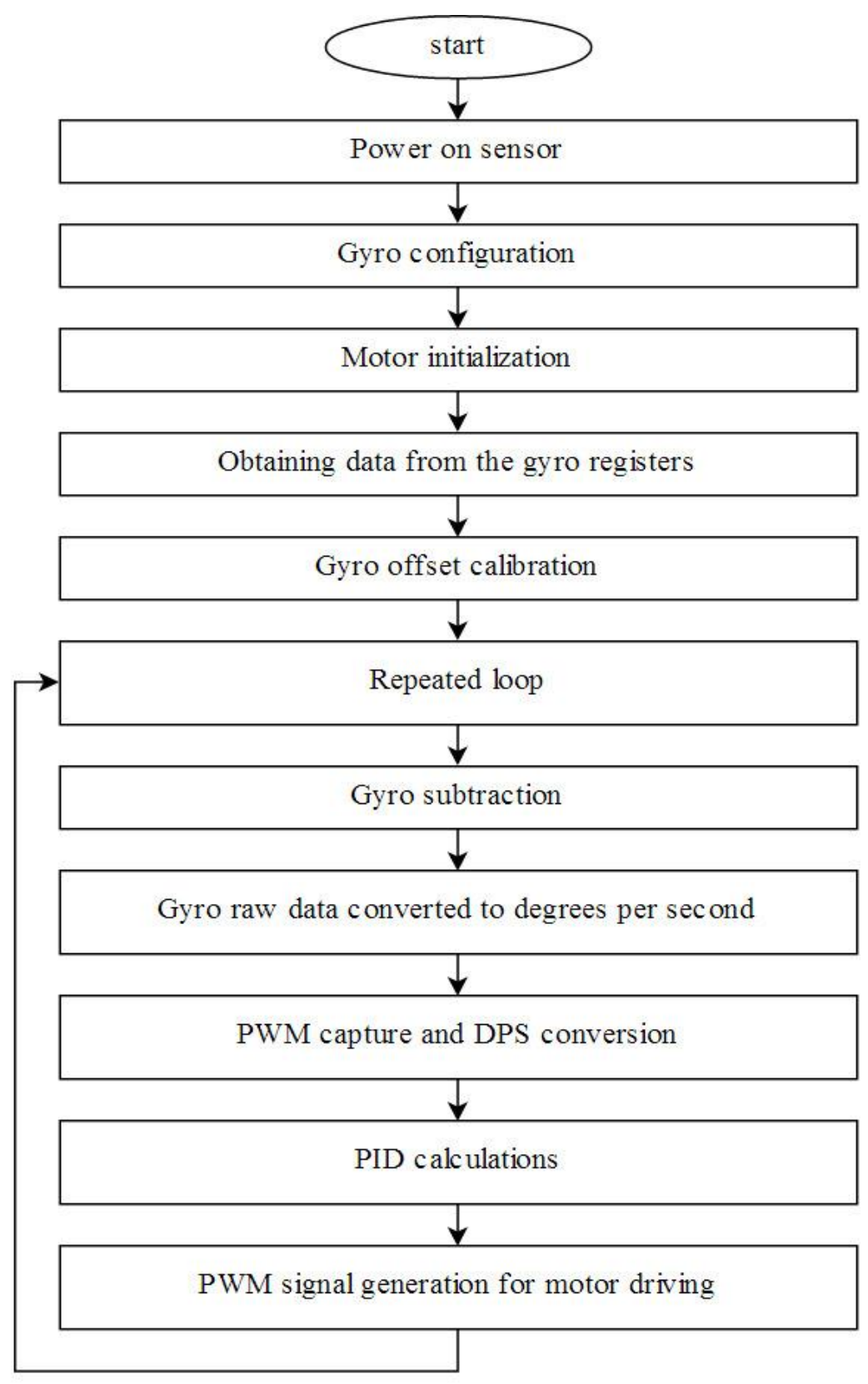

Fig 5: Implementation flow

a. Generation of PWM signals through the code.

b. Generation of PWM signals at the required rate i.e. $50 \mathrm{~Hz}$ frequency and 1$2 \mathrm{msec}$ time interval through the code.

c. Driving the BLDC motors through the PWM signal and testing the speed through the variation of the values given in the code.

d. In driving the BLDC motors we also test the variation in roll, yaw, throttle and pitch of the motor using the receiver and the transmitter hardware in simulation mode. 
e. Now the coding is done by using the triple timer counter of the Zed board so as to drive the four BLDC motors. Two motors in the clockwise direction and the other two in the anti-clockwise direction.

f. It is the code developed using the triple timer counter the speeds of the motors are altered and accordingly the flying motion of the Z-Quad changes. A lot of testing is done over here to check the proper functioning of the motors.

g. The Gyro and the Accelerometer hardware are configured and interfaced to the board using the embedded $\mathrm{C}$ coding. The gyro and the accelerometer give the position and the axis for the Z-Quad. It is here the roll, yaw, throttle and pitch of the Z-Quad is actually tested. Here we use I2C communication protocol.

h. The RC receiver decoding is done using the embedded C coding.

i. The stabilization the Z-Quad is achieved using the PID (Proportional, integral derivative) algorithm. It is a code used for finding errors especially the differences between Gyro and RC receiver values. The values of both old and new are added and averaged from time to time. A lot of testing is done in this stage.

j. $\quad$ There is also a need for complimentary filtering for removing errors in the gyro.

k. The stage is set for Z-Quad to fly. In the test flights a lot of crashes took place and we had to replace the components especially the propellers and sometimes the motors gave in too.

1. After many successful flight operations the other components are added.

$\mathrm{m}$. The camera is added so as to give us the video footage of the area under consideration.

n. The camera is interfaced to the control unit of the ground station. This helps a lot in the Precision Agriculture. The camera could also be a thermal camera that gives us the picture of the entire crop under surveillance.

\section{EXPERIMENTAL RESULTS}

In our experimental set up we had the following results. After testing all the implementation procedures the Z-Quad is ready to fly. We had to go to a playground in order to experiment the prototype. At first trimming is done so as to stabilize the ZQuad in its flight. The takeoff was smooth. The experimental results matched the tested results. The flight time was for 8-10 minutes. For the video coverage results the following resulted. The Cortex-A9 processors executed the ARMv7-A instruction set. The high-performance scalar floating-point computation single instruction multiple data (SIMD) Neon Media Processing Engine with which it is equipped with, is suitable for many applications [10][12]. The image processing algorithms are executed at a very high rate because of the advanced SIMD instructions. The performance is accelerated because of the dual ARM and Neon. 


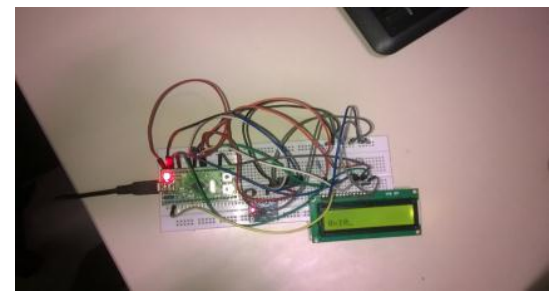

Fig 6: Interfacing of MPU 6050 sensor

The Neon has special processing engine registers. The most significant characteristic of its instruction set is its unique parallel mechanism. It has two input registers $\mathrm{Q}_{\mathrm{A}}$ and

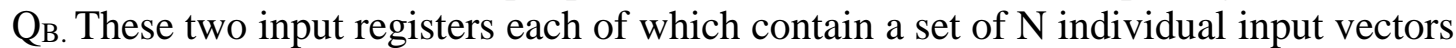
[13]. A single defined operation is performed between the $\mathrm{N}$ sets of input vectors to produce a corresponding set of output vectors which are written to the output register Qc. The routes are independent of each other. These can produce results at the same time with several different sets of inputs [10]. Each Neon MPE register loads 128 bit data directly from the DDR through the AXI bus. Then the data is processed and finally it is stored. All operations are performed through programming in the $\mathrm{C}$ language thus achieving the optimal processing efficiency.

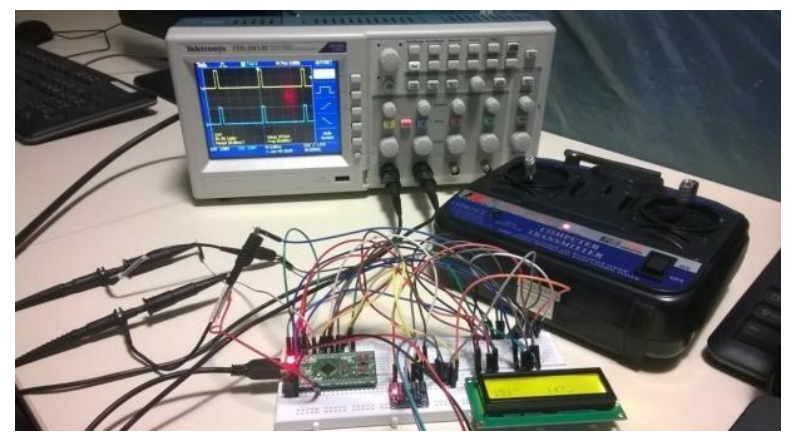

Fig 7: PWM signal generation using Triple Timer Counter

In order to improve the efficiency of system development, the Intel OpenCV library is used. The FPGA logic fabric permits a high-speed, fully parallel version of the coprocessor to be implemented. It is a hand-coded RTL design. The accelerators are generated to take a $\mathrm{C}$ description of the algorithm using Vivado high level synthesis (HLS) tools. The Vivado HLS tools generate Gauss filter IP and are thus attached on the AXI bus. ARM0 is given the object tracking and calculation of Kalman filter as the amount of calculation is very small. HDMI exports the results of detection and tracking. The Xilinx Zed board has 512Mb RAM attached and Dual ARM Cortex-A9 hard-core processor system (PS) running on $667 \mathrm{MHz}$. The logic (PL) IP is implemented with $100 \mathrm{MHz}$ clock frequency. The Image data is sent from PC to the SD card memory through Enet. The size of each frame is $1137 \times 686$. The time 
performance is 768 milliseconds per frame, which includes data flow, IP hardware execution and Neon acceleration. The algorithm is implemented through software.

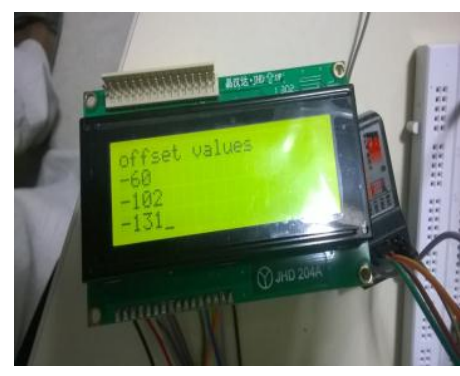

Fig 8: Gyro Offset Values

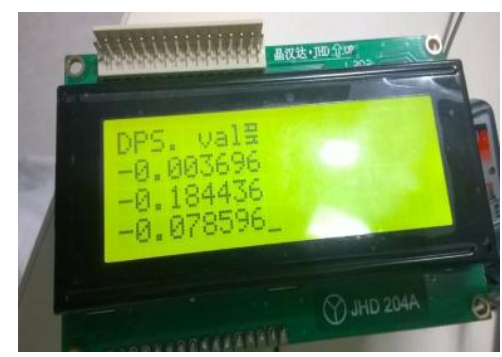

Fig 10: Gyro DPS Values

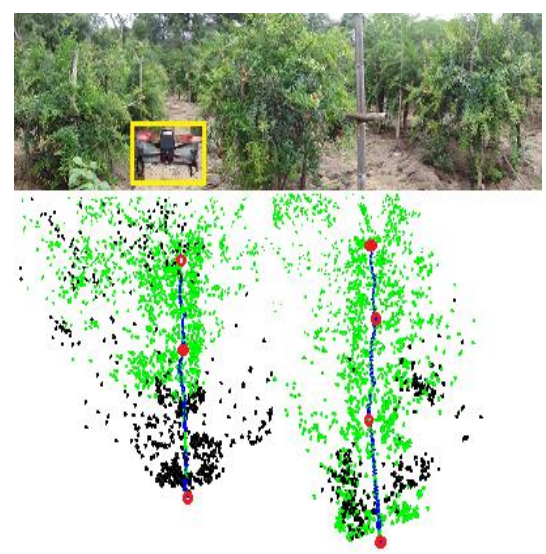

Fig 12: Estimating the Crop

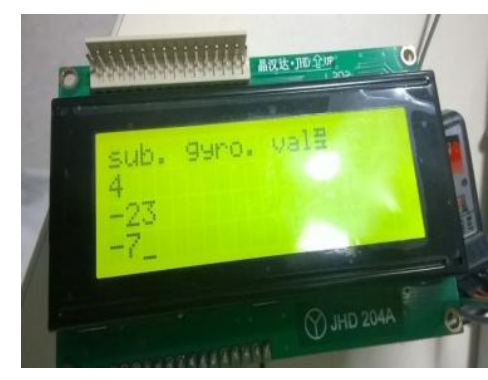

Fig 9: Gyro Subtraction Values

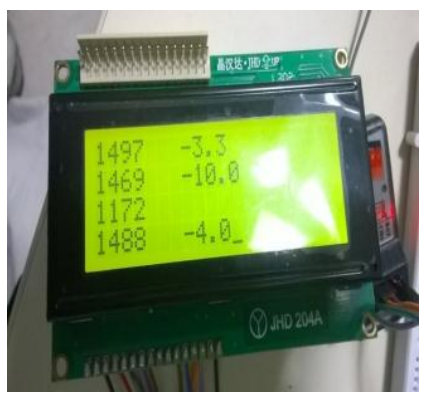

Fig 11: Receiver PWM captured signals and their DPS values.

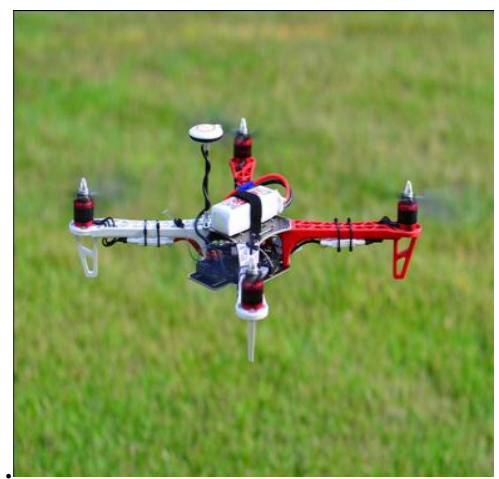

Fig 13: Z-Quad in Surveillance mode.

\section{CONCLUSION \& FUTURE WORK}

In this paper, I have given the architecture of the prototype Z-Quad with all its specifications, applications, hardware and software components. Every step in the implementation process took a lot of time. The prototype is ready to fly but given to the extreme heat conditions in my environment there are certain obstacles in the performance of the flight. Besides these constraints the cost for the purchase of the 
standard components has been on the rise. Thanks to the Centre for Development of Advanced Computing that has given me a platform to do this work. The main advantage in using Zed board in this particular prototype is the object detection and tracking system that combines hardware and software co-design which is implemented on Xilinx's Zed Board. It is very much necessary in the Precision Agriculture. This has a lot of advantages over other platforms in terms of the speed, power consumption, development difficulty and cost. The prototype is already flying but is yet to be tested in the Agriculture land with prior permissions from the authority.

The Future scope of this work relies very much on increasing the flight time of the ZQuad. The power consumed by the Z-Quad has to be charged in its flight time itself. Interfacing solar panels to recharge the battery on the Z-Quad is the only solution available to increase the flight time of the Z-Quad. All said and done many more features like the Wi-Fi, remote sensors and hazardous mission possible features can be implemented. With all these features the weight increases and accordingly the capacity of the BLDC motors also increases. All this opens up a big scope for the future work on the Z-Quad.

\section{REFERENCES}

[1] Zynq-7000 AP SoC Technical Reference Manual, UG585 (v1.10) February 23, 2015, http://www.xilinx.com/, pp 26-245

[2] Blosch, M.; Weiss, S.; Scaramuzza, D.; Siegwart, R., "Vision based MAV navigation in unknown and unstructured environments," Robotics and Automation (ICRA), 2010 IEEE International Conference ,3-7 May 2010.

[3] Camera-Based Navigation of a Low-Cost Quadrocopter (J. Engel, J. Sturm, D. Cremers), In Proc. of the International Conference on Intelligent Robot Systems (IROS), 2012.

[4] Stephan Weiss, Markus W. Achtelik, Simon Lynen, Margarita Chli, Roland Siegwart, "Real-time onboard visual-inertial state estimation and selfcalibration of MAVs in unknown environments," Robotics and Automation (ICRA), 2012 IEEE International Conference pp.957, May 2012.

[5] Roberto G. Valenti, Ivan Dryanovski, Carlos Jaramillo, Daniel Perea Strom, Jizhong Xiao, "Autonomous Quadrotor Flight Using Onboard RGB-D Visual Odometry" in Proc. IEEE Intl. Conf. on Robotics and Automation (ICRA), 2014.

[6] M. Achtelik, S. Weiss, and R. Siegwart, "Onboard IMU and Monocular Vision Based Control for MAVs in Unknown In-and Outdoor Environments," in Proc. IEEE Intl. Conf. on Robotics and Automation (ICRA), 2011.

[7] Shaojie Shen, Yash Mulgaonkar, Nathan Michael, and Vijay Kumar, "MultiSensor Fusion for Robust Autonomous Flight in Indoor and Outdoor Environments with a Rotorcraft MAV" in Proc. IEEE International Conference on Robotics and Automation (ICRA), 2014. 
[8] Mario Bergeron (Avnet, Inc.), Steve Elzinga, Gabor Szedo, Greg Jewett, and Tom Hill (Xilinx, Inc.), "Application Note: Zynq-7000 All Programmable SoC Video and Imaging Kit- XAPP794 (v1.3)”, December 20, 2013.

[9] Vivado Hello World Tutorial, "Embedded Processor Hardware Design", September 9, 2013.

[10] Xilinx Inc. UG585, “Zynq-7000 extensible processing platform technical reference manual”, 2012.

[11] Georg Klein and David Murray, "Parallel Tracking and Mapping for Small AR Workspaces", International Symposium on Mixed and Augmented Reality (ISMAR'07, Nara).

[12] Xilinx Inc. Xapp1078, "Simple AMP running Linux and bare-metal system on both Zynq SoC processors", 2013.

[13] Xilinx Inc. Xapp890, "Zynq All Programmable SoC sobel filter implementation using the Vivado HLS tool". 
